\title{
Modeling of Statistical Low-Frequency Noise of Deep-Submicrometer MOSFETs
}

\author{
Gilson I. Wirth, Member, IEEE, Jeongwook Koh, Roberto da Silva, Roland Thewes, Member, IEEE, and \\ Ralf Brederlow
}

\begin{abstract}
The low-frequency noise (LF-noise) of deep-submicrometer MOSFETs is experimentally studied with special emphasis on yield relevant parameter scattering. A novel modeling approach is developed which includes detailed consideration of statistical effects. The model is based on device physics parameters which cause statistical variations in LF-noise behavior of individual devices. Discrete quantities are used and analytical results for the statistical parameters are derived. Analytical equations for average value and standard deviation of noise power are provided. The model is compatible with standard compact models used for circuit simulation.
\end{abstract}

Index Terms-Analog circuits, low-frequency noise (LF-noise), MOS transistors, noise modeling, RF circuits, semiconductor device noise.

\section{INTRODUCTION}

$\mathbf{L}$ OW-FREQUENCY noise (LF-noise) is a performance limiting factor in many of today's CMOS analog and RF circuits. Aiming for robust circuit design, it is essential to develop a detailed understanding of the devices' noise behavior.

Recent works show that the LF-noise performance of modern small area MOS devices is dominated by random telegraph signal (RTS) fluctuations [1]-[10]. Their origin is the capture and subsequent emission of charge carriers at discrete trap levels near the $\mathrm{Si} / \mathrm{SiO}_{2}$ interface. Noise performance may strongly vary between different devices on one chip, and moreover even between different operation points of a single device. Although LF-noise has deserved great attention, today, detailed statistical models are not available. Due to the even yield restricting effect of LF-noise in many applications (e.g., in wireless transceiver designs) the need to statistically model the noise behavior increases, in particular for future analog and RF products. This paper is aiming for provision of a comprehensive

Manuscript received October 27, 2004; revised April 14, 2005. The review of this paper was arranged by Editor M. J. Deen.

G. I. Wirth is with UFRGS, Porto Alegre, Brazil 91501-970. He is also with the State University of Rio Grande do Sul (UERGS), Guaíba RS 92500, Brazil (e-mail: gilson-wirth@uergs.edu.br).

R. da Silva is with the Informatics Institute, Federal University of Rio Grande do Sul (UFRGS), Porto Alegre RS 91501-970, Brazil.

J. Koh was with Corporate Research, Infineon Technologies, Munich D-81730, Germany. He is now with the Communication and Network Laboratory, Samsung Advanced Institute of Technology, Samsung Electronics, Kiehung K440600, Korea (e-mail: jeongwook.koh@samsung.com).

R. Thewes and R. Brederlow are with Corporate Research, Infineon Technologies, Munich D-81730, Germany.

Digital Object Identifier 10.1109/TED.2005.850955

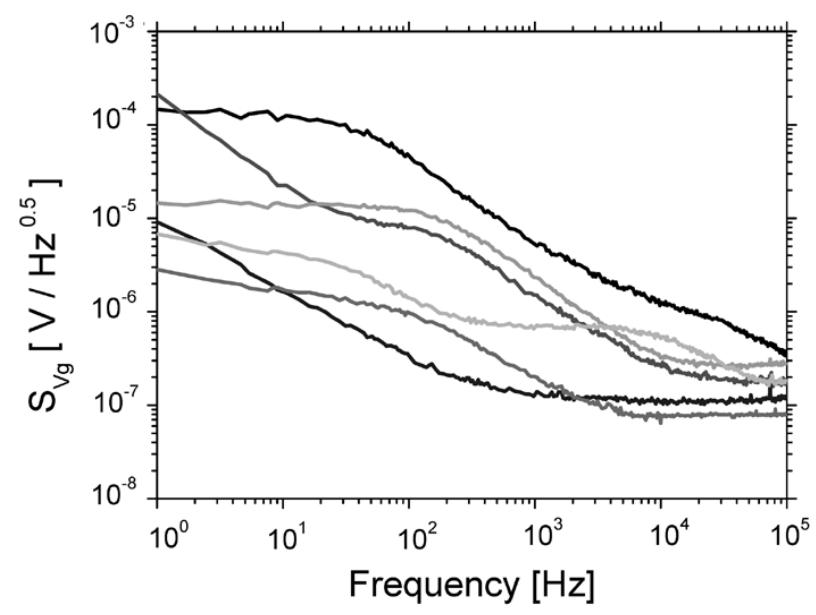

Fig. 1. Gate referred voltage noise of six different $W=0.16 \mu \mathrm{m} / L=$ $0.13 \mu \mathrm{m}$ n-MOS transistors from a $0.13-\mu \mathrm{m}$ standard CMOS process with $t_{\mathrm{ox}}=2.2 \mathrm{~nm}$ and $V_{\mathrm{th}}=300 \mathrm{mV}$. Characterization in saturation at $V_{G}=0.55 \mathrm{~V}$ and $V_{D}=1 \mathrm{~V}$.

understanding of the variations of LF-noise performance of deep-submicrometer devices.

The paper is organized as follows. After a brief discussion concerning the basics of RTS noise, a statistical modeling approach is presented based on the physical origin of the LF-noise in Section III. There, the dependence of noise performance on device geometry and operation point is studied in detail. The model is compared to experimental data from three different technology nodes $0.25\left(t_{\mathrm{ox}}=5 \mathrm{~nm}\right), 0.13\left(t_{\mathrm{ox}}=2.2 \mathrm{~nm}\right)$, and $0.09 \mu \mathrm{m}\left(t_{\mathrm{ox}}=1.6 \mathrm{~nm}\right)$ in Section IV. Finally, in Section V, the paper is concluded.

\section{RTS AND $1 / f$ NOISE}

In this section, the average LF-noise of individual MOSFETs is briefly reviewed, and some important parameters for the statistical evaluations following in Section III are introduced. The LF-noise of small area devices shows Lorentzian-like spectra as shown in Fig. 1. Strong variations are observed for the spectra of different devices with same geometries and from the same chip. In Fig. 2, the strong dependence of RTS noise on the bias point is shown. This behavior poses great challenges to design for high yield of minimal area low noise analog and RF circuits in advanced CMOS technologies. Deviations of orders of magnitude are observed between individual devices and at different operating points of a single device (cf. Figs. 1 and 2). 


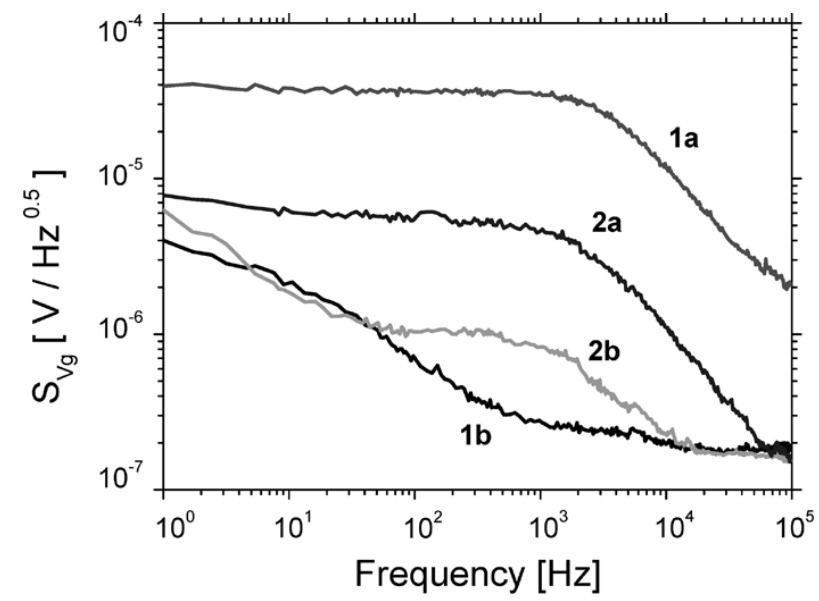

Fig. 2. Gate referred voltage noise of two different $W=0.16 \mu \mathrm{m} / L=$ $0.13 \mu \mathrm{m}$ n-MOS transistors from a $0.13-\mu \mathrm{m}$ standard CMOS process with $t_{\mathrm{ox}}=2.2 \mathrm{~nm}$ and $V_{\mathrm{th}}=300 \mathrm{mV}$ under different characterization conditions. Curves (1a) and (1b): first device biased at $V_{G}=0.85 \mathrm{~V}$, with $V_{D}=0.15 \mathrm{~V}$ and $V_{D}=1.0 \mathrm{~V}$, respectively; curves (2a) and (2b): second device, biased at $V_{D}=1.0 \mathrm{~V}$, with $V_{G}=0.85 \mathrm{~V}$ and $V_{G}=0.55 \mathrm{~V}$, respectively.

Noise spectra of today's small area devices are believed to be dominated at least for a certain frequency band by RTS from single-trap states. This assumption has been experimentally confirmed by time domain RTS measurements from several groups [2], [12].

As basis for the statistical modeling, the physics behind LF-noise phenomena are discussed here with special emphasis on their microscopic nature. Traps located in the gate oxide near the interface to the silicon capture and reemit some of the carriers responsible for the drain current flowing between source and drain of the device [2]-[6], [16]. The impact of the variation of the charging state of these traps on drain current has a similar effect as a fluctuation of the gate voltage. Therefore, an equivalent gate voltage fluctuation is frequently used to derive a simple equation for MOS LF-noise. In this paper, all experimental results are presented as equivalent gate voltage fluctuations.

To derive an approximation for the LF-noise we start with a simple equation for the drain current [20]

and

$$
I_{d}=q \cdot W \cdot \mu(y) \cdot N_{c}(y) \cdot \frac{\partial V(y)}{\partial y}
$$

$$
I_{d} \approx g_{m}\left(V_{g, \mathrm{eff}}\right) \cdot V_{g, \mathrm{eff}} .
$$

Here, $q$ is the elementary charge, $W$ the device width, $\mu(y)$ the mobility at location $y$ in the channel, $g_{m}$ the transconductance, $N_{c}(y)$ the local carrier density, $\partial V(y) / \partial y$ the local lateral electrical field in the transistor, and $V_{g, \text { eff }}$ is the effective gate-voltage, defined by the difference between gate-voltage and threshold voltage. $N_{c}(y)$ is given by the following equation [22]:

$$
N_{c}(y) \cong\left(V_{g, \text { eff }}-V(y)\right) \cdot C_{\text {ox }} / q .
$$

Here, $V(y)$ is the difference between channel potential at location $y$ and source-voltage, $C_{\mathrm{ox}}$ the gate capacitance per area. Equation (2) is normally used for transistor operating conditions in the linear mode [22], but it is also valid for transistor operating points under saturation conditions to describe the current in the channel region between source and pinch-off point. Since this is the by far most relevant region for $1 / f$-noise considerations it is suitable for our purposes in all cases.

For the effective gate voltage in subthreshold operating points, we use the following approximation known from the BSIM3 models to ensure a continuously differentiable function for the drain current formulation in weak inversion [23]:

$$
V_{g, \text { eff }}=2 \cdot n \cdot k T \frac{\ln \left(1+\exp \left(q V_{g, \text { eff }}^{\prime} / 2 n k T\right)\right)}{1+C_{\mathrm{ox}} \cdot \sqrt{\frac{2 \Psi_{s}}{q \varepsilon_{\mathrm{Si}} N_{\mathrm{ch}}}} \cdot \exp \left(q V_{g, \text { eff }}^{\prime} / 2 n k T\right)} .
$$

The term $k T$ is the thermal energy, $V_{g \text {,eff }}^{\prime}=V_{\mathrm{gs}}-V_{\mathrm{th}}$ is the effective gate voltage as applied to the terminals of the device and $V_{g, \text { eff }}$ is an equivalent effective gate voltage as it is inserted into the following equations. In strong inversion $\mathrm{V}_{g \text {, eff }}^{\prime}$ is similar

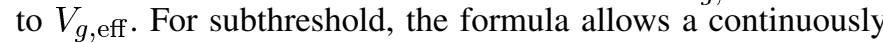
differentiable enhancement of the classical current formulation (1). A discussion of other parameters in (3), which are not important in this context, is given in [23]. Here, we use this formulation to align with the modeling quasi-standard. Equations (2) and (3) give us a drain current formula for deriving the standard deviation of the drain current (in other words, the current noise) due to trap influence. For simplicity, the reader may assume that $V_{g \text {,eff }}$ is equal to $V_{g \text {,eff }}^{\prime}$ in most practical cases.

The influence of the traps on the drain current is twofold. On the one hand, the occupation of a trap changes the number of free carriers in the channel, on the other hand, a charged trap state has a strong influence on the local mobility near to its position due to Coulomb scattering. Current fluctuations are calculated according to [11]

$$
\begin{aligned}
\frac{\delta I_{d}}{I_{d}} & =\frac{\delta N_{c}}{N_{c}(y)}+\frac{\delta \mu}{\mu(y)} \\
& =\left(\frac{1}{N_{c}(y)} \cdot \frac{\delta N_{c}}{\delta N_{t}}+\frac{1}{\mu(y)} \cdot \frac{\delta \mu}{\delta N_{t}}\right) \cdot \delta N_{t} .
\end{aligned}
$$

Here, $N_{t}$ is the trap density per volume and energy. $\delta N_{c} / \delta N_{t}$ is the change in the number of free carriers versus the number of occupied traps and $\delta \mu / \delta N_{t}$ describes the influence of a charged trap state on the mobility at location $y$.

According to [21] the first term of (4) is given by the following relation:

$$
\frac{\delta N_{c}}{\delta N_{t}}=\frac{q^{2} / k T \cdot N_{c}(y)}{C_{\mathrm{ox}}+C_{\mathrm{it}}+C_{d}+q^{2} / k T \cdot N_{c}(y)}
$$

where $C_{\text {it }}$ is the interface trap capacitance per area and $C_{d}$ the depletion capacitance per area of the MOS-structure (including pn-junction).

To derive an equation for the second term in (4) $\delta \mu / \delta N_{t}$ we have to approximate the influence of a trap on the local mobility. The mobility is given by the inverse sum of a Coulomb-scattering related term $\mu_{C}$, and an interface scattering related contribution $\mu_{\mathrm{SR}}$ [22]

$$
\frac{1}{\mu}=\frac{1}{\mu_{\mathrm{SR}}}+\frac{1}{\mu_{C}} .
$$


According to [24], interface scattering and coulomb-scattering are approximated by

and

$$
\mu_{\mathrm{SR}}=\frac{\mu_{\mathrm{SR}, 0}}{\theta\left(V_{g, \mathrm{eff}}-V(y)+Q_{B} / C_{\mathrm{ox}}\right)^{2}}
$$

$$
\mu_{C}=\frac{\mu_{0, C}}{\gamma \cdot N_{\mathrm{tr}}+\beta}
$$

respectively, with $\mu_{\mathrm{SR}, 0}, Q_{B}, \theta, \beta, \gamma$, and $\mu_{0, C}$ being technology-dependent physics-based constants. Here, for simplicity we assume these parameters to be constant. Equations (6)-(8) finally result in

$$
\mu(y) \cong \frac{\mu_{0}}{1+\theta^{*}\left(V_{g, \text { eff }}-V(y)\right)}
$$

with $\mu_{0}$ and $\theta^{*}$ being constants resulting from $\mu_{\mathrm{SR}, 0}, \theta, \gamma$, and $\beta$.

On the basis of this equation the term $\delta \mu / \delta N_{t}$ can be easily calculated

$$
\frac{\delta \mu}{\delta N_{t}}=\frac{\gamma \cdot \mu^{2}}{\mu_{0, C}} \cdot \frac{S}{W \cdot L} .
$$

Here, $L$ is the length of the transistor and the scattering parameter $S$ is introduced for modeling the influence on the mobility contribution of the surface roughness to the fluctuation. A detailed discussion is given in [25]. In a next step we calculate the impact of the change of the number of occupied traps on the drain current

$$
\begin{array}{r}
\frac{\delta I_{d}}{\delta N_{t}}=\frac{V_{g, \text { eff }} \cdot g_{m}}{N_{c}(y) \cdot \mu(y)}\left(\frac{N_{c}(y) \cdot \mu(y)}{k T \cdot(} C_{\mathrm{ox}}+C_{\mathrm{it}}+C_{d}\right) / q^{2}+N_{c}(y) \\
\left.+\frac{\gamma \cdot \mu(y)^{2} \cdot N_{c}(y) \cdot S}{\mu_{0, C} \cdot W \cdot L}\right) .
\end{array}
$$

Using (5), (10), and (11), we obtain for the gate voltage fluctuations

$$
\begin{aligned}
\frac{\delta V_{g}}{\delta N_{t}}=\frac{q}{W L \cdot C_{\mathrm{ox}}}\left(\frac{V_{g, \mathrm{eff}}}{V_{1}+V_{g, \mathrm{eff}}-V(y)}\right. & \\
& \left.+\frac{S^{*} \cdot V_{g, \mathrm{eff}}}{V_{2}+V_{g, \mathrm{eff}}-V(y)}\right) .
\end{aligned}
$$

There, $V_{1}, V_{2}$, and $S^{*}$ are given by

$$
\begin{aligned}
V_{1} & =\frac{k T}{q} \cdot \frac{C_{\mathrm{ox}}+C_{\mathrm{it}}}{C_{\mathrm{ox}}} \\
V_{2} & =1 / \theta^{*} \\
S^{*} & =-\frac{\mu_{0} \cdot \gamma}{\mu_{C, 0}} \cdot \frac{C_{\mathrm{ox}}}{q} \cdot \frac{S}{\theta^{*}} .
\end{aligned}
$$

Fluctuations in the number of occupied or nonoccupied trap states $\delta N_{t}$ are related to the Fermi-Dirac distribution $f(E)$ and the mean time constant $\tau(x, E)$ for a change in the occupation of the traps [20]

$$
\begin{aligned}
\delta N_{t}^{2}=N_{t}(x, E) \cdot f(E) & \cdot(1-f(E)) \\
& \cdot \frac{4 \cdot \tau(x, E)}{1+(2 \pi f \tau(x, E))^{2}} \delta x \delta y \delta z \delta E
\end{aligned}
$$

where $N_{t}(x, E)$ is the trap density per volume and energy. Using this equation and (12), we calculate the gate voltage-related noise $d S_{V g}\left(=\delta V_{g}^{2}\right)$ per area in the channel at location $y$ caused by traps with a distance $x$ from the interface and the energy $E$ at frequency $f$

$$
\begin{aligned}
d S_{V g} & (f, x, y, E) \\
= & \frac{q^{2}}{W^{2} L^{2} \cdot C_{\mathrm{ox}}^{2}} \cdot\left(\frac{V_{g, \mathrm{eff}}}{V_{1}+V_{g, \mathrm{eff}}-V(y)}\right. \\
& \left.+\frac{S^{*} \cdot V_{g, \mathrm{eff}}}{V_{2}+V_{g, \mathrm{eff}}-V(y)}\right)^{2} \\
& \cdot N_{t}(E, x) \cdot f(E, x)(1-f(E, x)) \\
& \cdot \frac{4 \cdot \tau(E, x)}{1+(2 \pi f \tau(E, x))^{2}} d E d x d y d z .
\end{aligned}
$$

Note that this approximation is somewhat different from previous formulations of that problem [2], [11], [20], [27] since we take into account local mobility effects at different locations within the device channel. For small area devices where the integration of trap and energy densities does not describe the correct behavior, we have to use a discrete summation instead of distributed quantities and the integrants $d E, d x, d y$, and $d z$

$$
S(f)=\sum_{i=1}^{N_{\mathrm{tr}}} A_{i}^{2} \frac{1}{f_{i}} \frac{1}{1+\left(\frac{f}{f_{i}}\right)^{2}}
$$

$N_{\text {tr }}$ is the number of traps in the active region of the device. The parameter $f_{i}$ defines the corner frequency of the Lorentzian spectrum of a discrete trap with index $i$

$$
f_{i}=1 / 2 \pi \tau(E, x) .
$$

In the following, statistical parameters for LF-noise behavior are derived on basis of (18) with $A_{i}$ summarizing a number of terms from (17)

$$
\begin{aligned}
A_{i}^{2}= & \frac{2 q^{2}}{\pi \cdot W^{2} L^{2} \cdot C_{\mathrm{ox}}^{2}}\left(\frac{V_{g, \mathrm{eff}}}{V_{1}+V_{g, \mathrm{eff}}-V\left(y_{i}\right)}\right. \\
& \left.+\frac{S^{*} \cdot V_{g, \mathrm{eff}}}{V_{2}+V_{g, \mathrm{eff}}-V\left(y_{i}\right)}\right)^{2} f\left(E_{i}, x_{i}\right) \\
& \times\left(1-f\left(E_{i}, x_{i}\right)\right) \cdot \Delta x_{i} \cdot \Delta y_{i} \cdot \Delta z_{i}
\end{aligned}
$$

$A_{i}$ is the Fourier transformation of the gate referred voltage amplitude of a single trap at position $x_{i}, y_{i}, z_{i}$ contributing to the noise. This formulation for $A_{i}$ is adequate for modeling approaches using distributed quantities.

Considering a larger number of small area devices, or the average behavior of small devices, the LF-noise can be calculated using continuously distributed quantities like trap densities instead of discrete ones. In the following, we briefly show the equivalence of the discrete and the density based $1 / f$ noise calculations. The current set of equations derived here leads to models similar to those well know from the literature when applied to large area considerations.

The integration of (17) leads to

$S_{V g}(f)=\frac{q^{2} k T N_{t}\left(E_{f}\right)}{4 \alpha C_{\mathrm{ox}}^{2} W L^{2}}$

$\cdot \frac{1}{f} \int\left(\frac{V_{g, \text { eff }}}{V_{1}+V_{g, \text { eff }}-V(y)}+\frac{S^{*} \cdot V_{g, \text { eff }}}{V_{2}+V_{g, \text { eff }}-V(y)}\right)^{2} d y$.

Here, $\alpha$ is the tunnel parameter assuming a WKB-like tunneling behavior of the traps, calculated similar to [20]. For the trap density per energy and per volume close to the Fermi level 
$N_{t}\left(E_{f}\right)$ we assumed a constant spatial distribution when performing the integration over $x$.

According to [22] the incremental location $d y$ is related to the incremental channel potential $d V$ by

$$
d y=\frac{W \cdot \mu_{0} \cdot C_{\mathrm{ox}} \cdot V_{2}}{g_{m} \cdot V_{g, \text { eff }}} \cdot \frac{V_{g, \text { eff }}-V(y)}{V_{2}+V_{g, \text { eff }}-V(y)} d V
$$

Since the traps between drain and pinch-off point only provide a negligible contribution to the transistors noise, the integration can be simplified by setting the pinch-off point as the upper integral boundary. Integration leads to a formulation of the LF-noise caused by trap states at the oxide-semiconductor interface

$$
\begin{aligned}
S_{V g}(f) & =S_{V g}^{0} \cdot \frac{V_{g, \text { eff }}}{g_{m}}\left[\frac { 1 } { V _ { 2 } } \left(\ln \left(\frac{V_{1}+V_{g, \text { eff }}}{V_{2}+V_{g, \text { eff }}} \frac{V_{2}+V_{g d}}{V_{1}+V_{g d}}\right)\right.\right. \\
& \left.+\frac{V_{1}}{V_{2}+V_{g, \text { eff }}}-\frac{V_{1}}{V_{2}+V_{g d}}\right) \\
& +2 S^{*}\left(\ln \left(\frac{V_{1}+V_{g d}}{V_{2}+V_{g d}} \frac{V_{2}+V_{g, \text { eff }}}{V_{1}+V_{g, \text { eff }}}\right)\right. \\
& \left.+\frac{1}{V_{2}+V_{g d}}-\frac{1}{V_{2}+V_{g, \text { eff }}}\right) \\
& \left.+\frac{S^{* 2}}{2}\left(\frac{V_{2}-2 V_{g d}}{\left(V_{2}+V_{g d}\right)^{2}}-\frac{V_{2}-2 V_{g, \text { eff }}}{\left(V_{2}+V_{g, \text { eff }}\right)^{2}}\right)\right] \cdot \frac{1}{f}
\end{aligned}
$$

with

$$
V_{g d}= \begin{cases}0, & \text { for } V_{g, \text { eff }} \leq V_{d} \\ V_{g, \text { eff }}-V_{d}, & \text { for } V_{g, \text { eff }}>V_{d}\end{cases}
$$

and

$$
S_{V g}^{0}=\frac{q^{2} k T N_{t}\left(E_{f}\right) \mu_{0}}{4 \alpha \theta^{*} C_{\mathrm{ox}} L^{2}}
$$

The model based on (23) is proven to be compatible to the compact models of the BSIM standard [26]. The model formulation is similar to the BSIM subthreshold formulation, but is continuous over the whole range of operating points of a MOSFET. For devices operating in inversion the main difference to BSIM and other analytical LF-noise models is the consideration of local differences in the mobility at different locations within the channel. This enables to eliminate one fit parameter compared to the BSIM approach [23], [27]. The remaining free parameters are the physics related fit parameters $N_{t}\left(E_{f}\right)$ and $\mu_{C, 0} / \gamma$, which describe number and mobility fluctuation related contributions to the LF-noise. All other parameters are standard BSIM parameters.

The model derived here includes a continuous formulation of the $1 / f$-noise behavior for all regions of operation as well as a reduction of the number of necessary fit parameters.

\section{Statistical LF-Noise Modeling}

The noise of a device itself is already a statistical parameter in time, namely the standard deviation of the drain current or, alternatively, of the equivalent gate voltage. To statistically model the variations of the noise when comparing different devices we have to identify the sources of noise voltage fluctuations. As can be seen from (18), the parameters sensitive to variations are the number of traps in the active region of the device $N_{\mathrm{tr}}$, the corner frequencies $f_{i}$ of the different traps, as well as the amplitude $A_{i}$ of the different traps. In the following, a description for the variance of each of these parameters is derived.

\section{A. Standard Deviation of the LF-Noise}

The number of traps $N_{\text {tr }}$ is assumed to follow a Poisson distribution. If $\left\langle N_{\mathrm{tr}}\right\rangle=N$ is the average number of traps per device in an ensemble of geometrically identical devices, the probability that $N_{\text {tr }}$ traps are found in a particular device is given by

$$
P\left(N_{\mathrm{tr}}\right)=\frac{N^{N_{\mathrm{tr}}} e^{-N}}{N_{\mathrm{tr}} !} .
$$

In order to roughly obtain a $1 / f$ spectrum, the time constants must be approximately uniformly distributed on a logarithmic scale [18], [19]. Since the average spectrum of large MOS devices roughly shows a $1 / f$-behavior it is reasonable to assume a similar distribution for the time constants. Physical processes that may lead to this distribution are, e.g., discussed in [19].

The probability distribution function of the trap corner frequency $f_{i}$ is then given by

$$
P\left(f_{i}\right)=\frac{1}{\ln \left(\frac{f_{\max }}{f_{\min }}\right)} \cdot \frac{1}{f_{i}} \cdot d f_{i} .
$$

The average number of traps $N$ is proportional to the active device area $W \times L$ and equal to

$$
N=N_{\text {dec }} \ln \left(\frac{f_{\max }}{f_{\min }}\right) W L .
$$

Here, $\left(N_{\text {dec }} \ln 10\right)$ is the trap density per unit area and frequency decade. The frequencies $f_{\min }$ and $f_{\max }$ delimit the frequency interval in which RTS is the origin of the LF-noise. $N$ is then the average number of traps with corner frequencies lying between $f_{\min }$ and $f_{\max }$.

In the next step, a noise model for the average noise of small area devices is developed based on statistical parameters of $A_{i}$.

The evaluation of the standard deviation of the average value of the noise power spectral density function $S(f)$ is

$$
\sigma_{S(f)}=\sqrt{\left\langle S_{f}^{2}\right\rangle-\left\langle S_{f}\right\rangle^{2}} .
$$

The average value of the noise power spectral density $\langle S(f)\rangle$ is evaluated by calculating the average value of (18) over $A_{i}, f_{i}$, and $N_{\text {tr }}$

$$
\left.\langle S(f)\rangle=\left\langle\left\langle\left\langle\sum_{i=1}^{N_{\mathrm{tr}}} A_{i}^{2} \frac{1}{f_{i}} \frac{1}{1+\left(\frac{f}{f_{i}}\right)^{2}}\right\rangle\right\rangle_{A_{i}}\right\rangle f_{f_{i}}\right\rangle .
$$

In the following $\left\langle A_{i}^{2}\right\rangle$ is written as $\left\langle A^{2}\right\rangle$. To evaluate the average over $f_{i}$, we use (27) for $P\left(f_{i}\right)$ leading to:

$$
\langle S(f)\rangle=\left\langle\sum_{i=1}^{N_{\mathrm{tr}}} \frac{\left\langle A^{2}\right\rangle}{\ln \left(\frac{f_{\max }}{f_{\min }}\right)} \int_{f_{\min }}^{f_{\max }} \frac{1}{f_{i}^{2}} \frac{\mathrm{df}_{i}}{1+\left(\frac{f}{f_{i}}\right)^{2}}\right\rangle
$$


After some mathematics we obtain

$$
\begin{aligned}
\langle S(f)\rangle & =\left\langle\sum_{i=1}^{N_{\mathrm{tr}}} \frac{\left\langle A^{2}\right\rangle}{\ln \left(\frac{f_{\max }}{f_{\min }}\right)}\right. \\
& \left.\times \frac{1}{f}\left(\arctan \left(\frac{f_{\max }}{f}\right)-\arctan \left(\frac{f_{\min }}{f}\right)\right)\right\rangle_{N_{\mathrm{tr}}}
\end{aligned}
$$

The evaluation of the average over $N_{\mathrm{tr}}$ using Poisson statistics leads to

$$
\langle S(f)\rangle=\frac{\left\langle A^{2}\right\rangle N_{\mathrm{dec}} W L}{f}\left(\arctan \left(\frac{f_{\max }}{f}\right)-\arctan \left(\frac{f_{\min }}{f}\right)\right) .
$$

If $f_{\min }$ is much smaller than the lower frequency at which the $1 / f$ noise is of practical interest, and if $f_{\max }$ is much higher than the frequency at which the thermal noise supersedes $1 / f$ noise, this results in

$$
\langle S(f)\rangle=\frac{\left\langle A^{2}\right\rangle N_{\mathrm{dec}} \mathrm{WL}}{f} \frac{\pi}{2}
$$

where $\left\langle A_{i}^{2}\right\rangle=\left\langle A^{2}\right\rangle$ is the average of the squared RTS amplitudes. This equation shows the commonly known $1 / f$ behavior. ${ }^{1}$

Note that proportionality to $1 / W L$ and to the average number of traps is obtained here similar to (23). This is because the average number of traps $\left\langle N_{\mathrm{tr}}\right\rangle=N$ in the device is proportional to $N_{\mathrm{dec}} \times W \times L$ and because $A$ is proportional to $1 / W L$. The equation above can be rewritten

$$
\langle S(f)\rangle=\frac{k f_{1}\left(V_{d}, V_{g}\right) N}{\mathrm{WL} f}
$$

Here, $f_{1}\left(V_{\mathrm{d}}, V_{g}\right)$ contains the bias point dependence hidden in the parameter $\left\langle A^{2}\right\rangle$ in the above equation and $k$ is a constant. Therefore the final result is equivalent to (23) and therefore also to the standard LF-noise models used for BSIM formulation [27]. The main difference is the use of a microscopic formulation of the LF-noise which helps determining its statistical behavior. As already mentioned this derivation does not detail the dependence of the LF-noise on the operating point, ${ }^{2}$ but shows that the statistical approach used here is equivalent to the results for the average noise of large area devices [see (23)]. Note that this is not a shortcoming of this formulation, but it is a problem which is of minor importance here, since variations in noise behavior are much higher than the dependence of the average value on bias point. Moreover, the dependence of the fluctuations in amplitude on bias point will be properly modeled in the next section (55).

\footnotetext{
${ }^{1}$ The reader is strongly encouraged to contact the authors on the details of the derivations of the model equations. To explicitly show all derivations would lead to an inappropriately large amount of equations and will be published elsewhere.

${ }^{2}$ This dependence is hidden in the parameter $\left\langle A^{2}\right\rangle$ in (34), and $A$ is given by (40).
}

Next for calculating the standard deviation, we need to calculate $\left\langle S(f)^{2}\right\rangle$. We start with

$$
\begin{aligned}
\left\langle S(f)^{2}\right\rangle & =\left\langle\left\langle\left\langle\sum_{i=1}^{N_{\mathrm{tr}}} \frac{A_{i}^{4}}{f_{i}^{2}} \frac{1}{\left(1+\left(\frac{f}{f_{i}}\right)^{2}\right)^{2}}\right.\right.\right. \\
& \left.\left.+\sum_{i \neq \mathrm{j}}^{N_{\mathrm{tr}}} \frac{A_{i}^{2} A_{\mathrm{j}}^{2}}{f_{i} f_{\mathrm{j}}} \frac{1}{1+\left(\frac{f}{f_{i}}\right)^{2}} \frac{1}{1+\left(\frac{f}{f_{\mathrm{j}}}\right)^{2}}\right\rangle_{A_{i}}\right\rangle_{f_{i} N_{\mathrm{tr}}} .
\end{aligned}
$$

The evaluation of the above equation leads to the standard deviation of the noise spectral density function $S(f)$ due to scattering of the parameters $A_{i}, f_{i}$, and $N_{\mathrm{tr}}$

$$
\sigma_{S(f)}^{2}=\frac{\left\langle A^{4}\right\rangle N_{\mathrm{dec}} \mathrm{WL}}{2}\left(\frac{1}{f_{\min }^{2}+f^{2}}-\frac{1}{f_{\max }^{2}+f^{2}}\right) .
$$

where $\left\langle A_{i}^{4}\right\rangle=\left\langle A^{4}\right\rangle$. If $f_{\min }$ becomes very small and $f_{\max }$ relatively large compared to the noise bandwidth of interest, a simplification is possible

$$
\sigma_{S(f)}^{2}=\frac{\left\langle A^{4}\right\rangle N_{\mathrm{dec}} \mathrm{WL}}{2} \frac{1}{f^{2}}
$$

The normalized standard deviation amounts to

$$
\frac{\sigma_{S(f)}}{\langle S(f)\rangle}=\frac{\sqrt{2}}{\pi \sqrt{N_{\mathrm{dec}} \mathrm{WL}}} \sqrt{\frac{\left\langle A^{4}\right\rangle}{\left\langle A^{2}\right\rangle^{2}}}
$$

Here, the contributions due to scattering of the parameters $A_{i}, N_{\mathrm{tr}}$, and $f_{i}$ are all taken into account.

As can be seen from (38) and (39), variations in the amplitude of individual RTS have a strong influence on the standard deviation of the LF-noise. Furthermore, from the parameters in (38) and (39) only $A$ may show the strong bias point dependence needed to explain the LF-noise behavior of small area devices. Hence, the sources of fluctuations in the amplitude of individual RTS and his dependence on bias point have to be investigated in more detail. This is done in the next section.

\section{B. Statistical Parameters of the LF-Noise Amplitude}

The amplitude $A_{i}$ of the $i$ th RTS power spectrum depends on the amplitude of the current fluctuation $\delta I_{d}$ as well as on the relation between the electron capture $\left(\tau_{c}\right)$ and emission $\left(\tau_{e}\right)$ time constants after [29]

$$
A_{i}^{2}=\delta \mathrm{I}_{\mathrm{d}}^{2} \frac{\beta}{(1+\beta)^{2}}
$$

with $\beta=\tau_{c} / \tau_{e}$. The current fluctuation $\delta I_{d}$ results from the combined effect of carrier number and mobility fluctuation as given by (4). The above formulation for $A_{i}$ is adequate for modeling approaches when using discrete quantities, in contrast to (20), which is adequate for modeling purposes when using distributed quantities. The formulation as given by (40) will be used in the remaining of this paper. 
From the definition of the standard deviation $\sigma_{A^{2}}=$ $\sqrt{\left\langle A^{4}\right\rangle-\left\langle A^{2}\right\rangle^{2}}$, it follows that

$$
\frac{\left\langle A^{4}\right\rangle}{\left\langle A^{2}\right\rangle^{2}}=1+\frac{\left(\sigma_{A^{2}}\right)^{2}}{\left\langle A^{2}\right\rangle^{2}} .
$$

The standard deviation of $A^{2}\left(\delta \mu / \mu, \delta N_{C} / N_{C}, \beta\right)$ is given by

$$
\begin{aligned}
\left(\sigma_{A^{2}}\right)^{2}= & \left(\frac{\partial A^{2}}{\partial(\delta \mu / \mu)}\right)^{2} \sigma_{\delta \mu / \mu}^{2} \\
& +\left(\frac{\partial A^{2}}{\partial\left(\delta N_{C} / N_{C}\right)}\right)^{2} \sigma_{\delta N_{C} / N_{C}}^{2}+\left(\frac{\partial A^{2}}{\partial \beta}\right)^{2} \sigma_{\beta}^{2}
\end{aligned}
$$

where $N_{c}(y)$ is the local carrier density as given by (2).

To model the total standard deviation of the noise power spectral density it is necessary to investigate the factors that influence mobility fluctuations $\delta \mu / \mu(y)$, carrier number fluctuations $\delta N_{C} / N_{C}(y)$ and fluctuations in the capture and emission time constant $\beta$.

We first investigate the mobility fluctuation term $\delta \mu / \mu(y)$. The mobility is impacted by carrier scattering at the location of the traps [cf. (10)]. Scattering efficiency depends on inversion layer parameters, like charge carrier velocity and carrier density, and on the device geometry. A charge closer to the interface scatters carriers more effectively than one further away [13]. If the vertical distance $d$ of the trap from the inversion layer is a random variable, it contributes to dispersion of the noise. To best of our knowledge analytical models for the scattering efficiency as a function of $d$ have not been published so far.

A reasonable first-order approach is to assume the scattering efficiency to be proportional to the intersection between the channel plane and the sphere defined by the critical trap radius $r_{c}$, as depicted in Fig. 3. The parameter $r_{c}$ is assumed to be either the distance of Coulomb interaction energy which is greater than $k T$ or the screening length $L_{S}$. Since the Coulomb potential is

$$
V(r)=q /(4 \pi \varepsilon r)
$$

with $r$ being the radial distance from the trap, the critical radius for Coulomb interaction $r_{\mathrm{kt}}$ is given by

$$
r_{\mathrm{kt}}=\frac{\mathrm{q}^{2}}{4 \pi \varepsilon \mathrm{kT} \mathrm{T}_{e}} .
$$

For a two-dimensional electron gas, $L_{S}$ is approximated by [12]

$$
L_{s}=\sqrt{2} \frac{\varepsilon \mathrm{kT}}{q N_{C}(y)} .
$$

In weak inversion, the channel carriers are scattered by the Coulomb potential. As the number of charge carriers increases screening of the Coulomb potential takes place, and the critical radius $r_{c}$ is given by the smallest of $L_{S}$ and $r_{k t}$ [12]. The radius of intersection between the channel plane and the sphere defined by the critical trap radius $r_{c}$ is

$$
r_{i}=\sqrt{r_{c}^{2}-d^{2}} .
$$

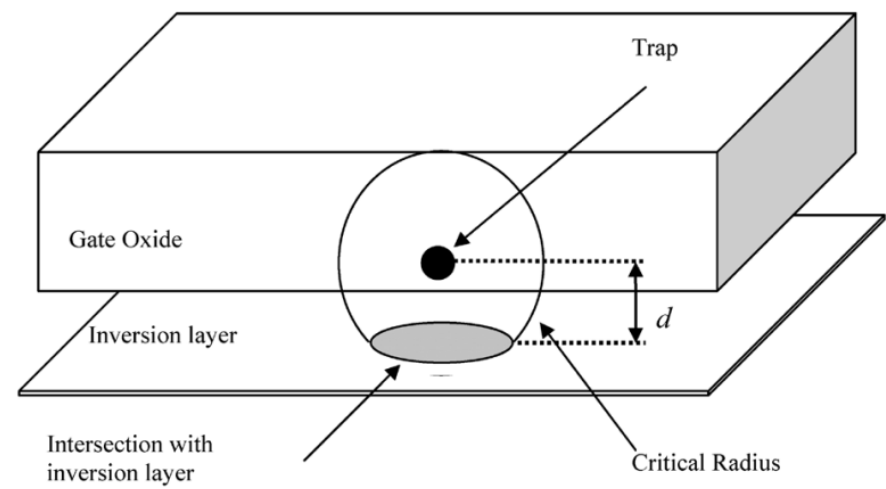

Fig. 3. Schematic plot of the inversion layer of a MOS transistor disturbed by an occupied trap state. From elementary geometry considerations, the radius $r_{i}$ of the intersection between the channel plane (inversion layer) and the sphere defined by the critical radius $r_{c}$ is calculated according to (46), for $d<r_{c}$. If $d \geq r_{c}$, it is assumed that the trap causes no scattering $(\delta \mu=0)$.

The channel area perturbed by the trap is then given by

$$
A_{\text {trap }}=\pi r_{i}^{2}=\pi\left(r_{c}^{2}-d^{2}\right) .
$$

Since Coulomb interaction and screening length both depend on inversion layer carrier concentration and carrier temperature $r_{c}$ and $A_{\text {trap }}$ may strongly depend on the bias point, especially at the drain side.

In a first-order approach $\delta \mu / \mu$ is estimated to be equal to the ratio between the perturbed area and the active channel area $(W \times L)$

$$
\frac{\delta \mu}{\mu(y)}=\frac{A_{\text {trap }}}{W L}=\frac{\pi r_{i}^{2}}{W L} .
$$

It is assumed that $d$ only affects the scattering efficiency of the trap, i.e., the change in mobility $\delta \mu$. There is no correlation between $d$ and $\delta N$ as long as $d \ll t_{\text {ox }}$. Since this condition is true for all traps with significant contributions to the device noise, this means that we can treat mobility and number fluctuations for different devices as statistically independent parameters.

The value of $r_{c}$ and hence $r_{i}$ depend on the bias conditions in a complex manner. Consequently, it is difficult to provide a closed expression for the variance in noise power due to mobility fluctuations. Nevertheless, at small drain bias $r_{c}$ can be assumed to be constant at all channel positions in a first-order approximation. If in addition all distances $d$ are assumed to have equal probabilities (for $0 \leq d \leq r_{c}$ ), then using (48)

$$
\begin{aligned}
\left\langle\frac{\delta \mu}{\mu}\right\rangle & =\left\langle\pi\left(\frac{\mathrm{r}_{\mathrm{c}}^{2}-\mathrm{d}^{2}}{\mathrm{WL}}\right)\right\rangle \\
& =\frac{\pi}{\mathrm{WL}} \int_{0}^{\mathrm{r}_{\mathrm{c}}} \frac{\mathrm{r}_{\mathrm{c}}^{2}-\mathrm{d}^{2}}{\mathrm{r}_{\mathrm{c}}} d \mathrm{~d}=\frac{2 \pi \mathrm{r}_{\mathrm{c}}^{2}}{3 \mathrm{WL}}
\end{aligned}
$$

and

$$
\begin{aligned}
\left\langle\left(\frac{\delta \mu}{\mu}\right)^{2}\right\rangle & =\left\langle\left(\frac{\pi\left(\mathrm{r}_{\mathrm{c}}^{2}-\mathrm{d}^{2}\right)}{\mathrm{WL}}\right)^{2}\right\rangle \\
& =\left(\frac{\pi}{\mathrm{WL}}\right)^{2} \int_{0}^{\mathrm{r}_{\mathrm{c}}} \frac{\left(\mathrm{r}_{\mathrm{c}}^{2}-\mathrm{d}^{2}\right)^{2}}{\mathrm{r}_{\mathrm{c}}} d \mathrm{~d}=\frac{8}{15}\left(\frac{\pi \mathrm{r}_{\mathrm{c}}^{2}}{\mathrm{WL}}\right)^{2} .
\end{aligned}
$$


Hence, $\sigma(\delta \mu / \mu) /\langle\delta \mu / \mu\rangle$ can be calculated from the definition of the standard deviation as

$$
\frac{\sigma(\delta \mu / \mu)}{\langle\delta \mu / \mu\rangle}=\frac{1}{\sqrt{5}}=\mathrm{k}_{\mathrm{d}} .
$$

The contribution of the mobility fluctuation to the total variance in noise power due to carrier scattering is particularly important at small drain voltages. Here, the channel is homogeneous and variance in $\delta N_{C} / N_{C}(y)$ approaches zero. Therefore, the variance due to mobility fluctuations can be modeled as a constant in a first-order approximation.

Let us now investigate the carrier number fluctuation term $\delta N_{C} / N_{C}(y)$. For this purpose, the carrier density $N_{C}(y)$ at all positions $y$ within the channel has to be known. This parameter depends on the bias condition and is a function of the local channel potential $V(y)$ within the channel, as given by (2).

Since the number of free carriers is a function of the position $y$, also the carrier number fluctuation term $\delta N_{C} / N_{C}(y)$ depends on $y$. The influence of a trap on the total current through the device depends on the local number of free carriers. Therefore, also the RTS amplitude depends on the position $y$ of a trap within the channel. At low drain voltage $V_{d}$ there is almost no dependence on $y$, but for transistor operating points in the saturation region this effect is strongly pronounced. For small drain voltages the carrier density is approximately homogenous within the whole channel. At high $V_{d}$ however, the carrier density decreases from source to drain, and $\delta N_{C} / N_{C}(y)$ increases from source to the pinch-off point, where it reaches its maximum. Beyond the pinch-off point and close to the devices drain the noise efficiency of a trap is negligible, since free carriers reach saturation velocity and there is no attractive field for free carriers at the interface. The scattering in RTS amplitudes due to the variance of $\delta N_{C} / N_{C}(y)$ increases with increasing drain bias and reaches a maximum when the device is operated in saturation.

In order to evaluate $\delta N_{C} / N_{C}(y)$ at different bias points a noise efficiency term $h(y)$ is introduced which describes the efficiency of a trap in producing noise related to number fluctuation [1]. The amplitude $A_{i}$ of the trap is then directly proportional to $h(y)$. This term depends on bias point and on the trap position, and is given by the following approximation:

$$
h(y)=\frac{\partial S_{V_{g}}(y)}{S_{V_{g}}}=\mathrm{K}_{y} \frac{V_{g, \mathrm{eff}}}{k \mathrm{~T} / \mathrm{q} \cdot\left(C_{\mathrm{ox}}+C_{\mathrm{it}}\right) / C_{\mathrm{ox}}+V_{\mathrm{gd}}(y)}
$$

with $h(y)$ normalized according to

$$
\int_{0}^{L} h(y) \mathrm{dy}=1
$$

and $V_{g d}(y)$ given by (52), shown at the bottom of the page, with $V_{g \text {,eff,krit }}=v_{s} L / \mu$ and $v_{s}$ being the saturation velocity. For simplicity, the potential $V(y)$ determining the local channel carrier concentration of the MOSFET is approximated by a linear fit here

$$
V(y)=V_{g, \mathrm{eff}} \cdot \frac{L-y}{L}+V_{d} \cdot \frac{y}{L} .
$$

In (50)-(52), we also take into account the effects arising from velocity saturation in the channel. If the vertical field at a certain position in the channel is sufficiently high for free carriers to reach saturation velocity in the inversion region, their density between this point and the drain junction remains constant. Within this region, $h(y)$ remains constant. Finally, near drain and beyond the pinch-off point there is no attractive field for free carriers at the interface, so that they do not interact with traps. For this reason the impact of traps in this region on trap related noise can be neglected.

Using these approximations we can calculate the dependence of $A_{i}$ on bias point. To get an expression for the standard deviation we have to statistically sum up the infinitesimal small areas with different amplitudes $A_{i}$ to the total noise and evaluate the resulting deviation. The resulting deviation of the noise amplitude from its average value is proportional to the integral of $h(y)^{2}$ from source to drain. Neglecting the nondominant terms in this integral, the normalized deviation of noise amplitude from its average amounts to [1]

$$
\frac{\sigma\left(\delta N_{C} / N_{C}\right)^{2}}{\left\langle\delta N_{C} / N_{C}\right\rangle^{2}} \approx \frac{V_{d}^{5}}{V_{g, \text { eff }}^{5}} .
$$

This equation describes statistical noise deviations due to the bias point. It neither depends on technology parameters or on device geometry nor requires additional fit parameters. For very small values of $V_{d}$ the density of carriers is homogenous along the channel, and $h(y)=1$ within the whole channel. This condition gives us minimal variation of the noise power spectral density. As $V_{d}$ increases the number of free carriers decreases with $y$ increasing, and $h(y)$ increases from source to drain resulting in higher variations of the noise power spectral density.

Finally, let us investigate the influence of capture and emission time constant variations $(\beta)$ on RTS amplitude fluctuations. When $\tau_{c}$ is equal to $\tau_{e}$ the corresponding RTS amplitude will be the largest. For asymmetrical RTS the amplitude will be smaller. Hence, the factor $\beta$ will introduce further variance in RTS noise amplitude $A$.

In order to evaluate $\sigma(\beta)$ analytically the exact bias point dependence of $\tau_{c}$ and $\tau_{e}$ is needed, and detailed time-domain parameter extraction of RTS is mandatory [29]. This will be the subject of future work. The contribution of scattering in $\beta$ to the standard deviation is proposed to be modeled as a constant, $k_{\beta}$, in a first-order approximation. If more information on the behavior of $\tau_{c}$ and $\tau_{e}$ is made available, the modeling of the contribution of scattering in $\beta$ to the standard deviation can be further improved.

$$
V_{g d}(y)= \begin{cases}0, & \text { for } V_{g, \mathrm{eff}, \mathrm{krit}}, \leq V_{d} \text { and } V_{g, \mathrm{eff}, \mathrm{krit}}>V_{g, \mathrm{eff}} \\ V_{g, \mathrm{eff}}-V_{g, \mathrm{eff}, \mathrm{krit}}, & \text { for } V_{g, \mathrm{eff}, \mathrm{krit}} \leq V_{d} \text { and } V_{g, \mathrm{eff}, \mathrm{krit}}<V_{g, \mathrm{eff}} \\ V_{g, \mathrm{eff}}-V(y), & \text { for } V_{g, \mathrm{eff}, \mathrm{krit}}>V_{d}\end{cases}
$$


The analysis above and experimental data show that the amplitude variations have a component that increases with bias point, as well as a component that is present even at small bias.

Based on the above discussions, (41), and [1], a simplified first-order approximation to fit experimental data is proposed

$$
\frac{\left\langle A^{4}\right\rangle}{\left\langle A^{2}\right\rangle^{2}}=1+\mathrm{k}_{\mathrm{a}} \frac{V_{d}^{5}}{V_{g, \text { eff }}^{5}}+\mathrm{k}_{\mathrm{b}}
$$

In the above equation, $k_{b}$ describes the variations in RTS amplitude that are present even at low drain bias, where $N_{C}(y)$ is homogenous within the whole channel and variations are due to scattering of $d$ and $\beta$ (arises from variations in $(\delta \mu / \mu)$ and variations in $\beta$, i.e., arises from $k_{d}$ and $\left.k_{\beta}\right)$. The term $k_{a} V_{\mathrm{d}}^{5} / V_{g, \text { eff }}^{5}$ weights the variations due to the nonhomogeneous contribution of the traps to the LF-noise depending on channel position at larger drain bias (arises from variations in $\left(\delta N_{C} / N_{C}\right)$ ).

A MiniMOS [17] device simulation is performed to investigate the term $\left\langle\delta I_{d}^{4}\right\rangle /\left\langle\delta I_{d}^{2}\right\rangle^{2}$ under different bias conditions. In the device simulations both number and mobility fluctuations are taken into account to determine the amplitude of the drain current fluctuations $\delta I_{d}$. Fig. 4 shows both device simulations and the results of $h(y)$, as a function of the trap position along the channel. Good agreement between the simplified model and the result from device simulations is found.

\section{Standard Deviation of LF-Noise for Different Bandwidths of Interest}

The noise amplitude at a given frequency $f$ and its standard deviation is an important parameter to the circuit designer. But also the noise power integrated over the circuit bandwidth, $n p_{\mathrm{BW}}$, and its related standard deviation are of interest in many cases. This parameter is given by the integration of (18) from $f_{L}$ to $f_{H}$, the lower and upper boundaries of the bandwidth of interest in a given circuit design

$$
n p_{\mathrm{BW}}=\int_{f_{L}}^{f_{\mathrm{H}}} S(f) \mathrm{df} .
$$

Inserting (18) in (56) leads to

$$
n p_{\mathrm{BW}}=\int_{f_{L}}^{f_{\mathrm{H}}}\left(\sum_{i=1}^{N_{\mathrm{tr}}} A_{i}^{2} \frac{1}{f_{i}} \frac{1}{1+\left(\frac{f}{f_{i}}\right)^{2}}\right) \mathrm{df} .
$$

The integral in the above equation can be solved to

$$
\begin{aligned}
n p_{\mathrm{BW}} & =\sum_{i=1}^{N_{\mathrm{tr}}} A_{i}^{2} \operatorname{arctg}\left(\frac{f_{\mathrm{H}}}{f_{i}}\right)-\sum_{i=1}^{N_{\mathrm{tr}}} A_{i}^{2} \operatorname{arctg}\left(\frac{f_{L}}{f_{i}}\right) \\
& =\sum_{i=1}^{N_{\mathrm{tr}}} n p_{i} .
\end{aligned}
$$

Here, $n p_{i}$ is the contribution of a single trap with corner frequency $f_{i}$ and amplitude $A_{i}$ to the noise power integrated over the bandwidth. The total noise power is the sum of the contribution of all traps. Notice that even if the corner frequency $f_{i}$

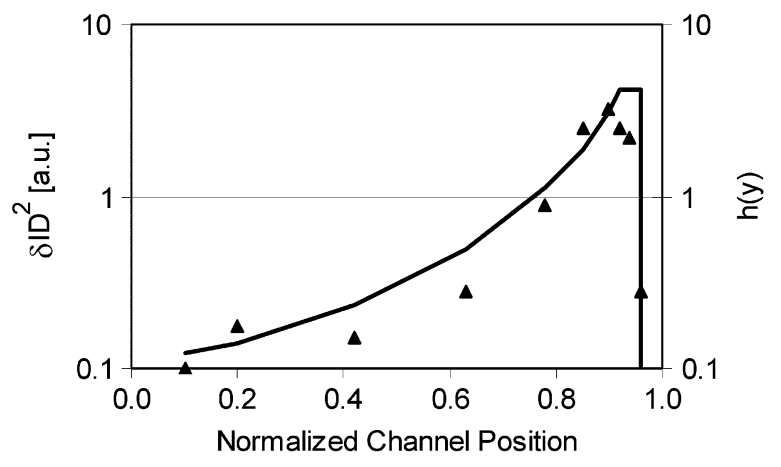

Fig. 4. Triangles: Simulated contribution from traps at different channel positions (source at $y=0$ and drain at $y=1$ ) on the trap related noise of a MOSFET operated in saturation (left axis). Full line: Efficiency term $h(y)$ as evaluated from (50) (right axis).

lies outside the bandwidth delimited by $f_{L}$ and $f_{H}$ it does contribute to the noise power in the bandwidth.

Both average value and standard deviation of a larger ensemble of nominally identically transistors (but with different statistically distributed traps) is evaluated. We start with the calculation of the average based on (58).

If $n p_{\mathrm{BW}}\left(N_{\mathrm{tr}}\right)$ is the noise power when the number of traps in the device is equal to $N_{\mathrm{tr}}$, and $P\left(N_{\mathrm{tr}}\right)$ is the probability that the number of traps in the device is equal to $N_{\mathrm{tr}}$, then

$$
\left\langle n p_{\mathrm{BW}}\right\rangle=\sum_{N_{\mathrm{tr}}=1}^{\infty} n p_{\mathrm{BW}}\left(N_{\mathrm{tr}}\right) P\left(N_{\mathrm{tr}}\right) .
$$

Here, $n p_{\mathrm{BW}}\left(N_{\mathrm{tr}}\right)$ is given by (57) and $P\left(N_{\mathrm{tr}}\right)$ follows a Poisson distribution. Hence

$$
\begin{aligned}
\left\langle n p_{\mathrm{BW}}\right\rangle & =\sum_{N_{\mathrm{tr}}=1}^{\infty} \sum_{i=1}^{N_{\mathrm{tr}}} n p_{i} \frac{N^{N_{\mathrm{tr}}} e^{-N}}{N_{\mathrm{tr}} !} \\
& =\sum_{N_{\mathrm{tr}}=1}^{\infty}\left(\left\langle n p_{i}\right\rangle N_{\mathrm{tr}}\right) \frac{N^{N_{\mathrm{tr}}} e^{-N}}{N_{\mathrm{tr}} !} .
\end{aligned}
$$

Let us first investigate the average of $n p_{i}$ given by

$$
\begin{aligned}
\left\langle n p_{i}\right\rangle= & \int_{f_{\min }}^{f_{\text {max }}} n p_{i}\left(A_{i}, f_{i}\right) \cdot P\left(f_{i}\right) \cdot \mathrm{df}_{i} \\
= & \frac{1}{\ln \left(\frac{f_{\max }}{f_{\min }}\right)} \\
& \times \int_{f_{\min }}^{f_{\max }}\left(A_{i}^{2} \operatorname{arctg}\left(\frac{f_{\mathrm{H}}}{f_{i}}\right)-A_{i}^{2} \operatorname{arctg}\left(\frac{f_{L}}{f_{i}}\right)\right) \frac{1}{f_{i}} d f_{i} .
\end{aligned}
$$

Here, $f_{\min }$ and $f_{\max }$ delimit the frequency interval in which RTS is the origin of the LF-noise. Note that those frequencies are different from $f_{L}$ and $f_{H}$, which are the boundaries of the bandwidth of interest in a given circuit design.

The evaluation of the above equation leads to

$$
\left\langle n p_{\mathrm{BW}}\right\rangle=\left\langle A^{2}\right\rangle \frac{\pi}{2} \ln \left(\frac{f_{\mathrm{H}}}{f_{L}}\right) N_{\mathrm{dec}} W L .
$$


In order to calculate the standard deviation we need to evaluate $\left\langle n p_{\mathrm{BW}}^{2}\right\rangle$ starting from

$$
\begin{aligned}
\left\langle n p_{\mathrm{BW}}^{2}\right\rangle & \left.\left.=\left\langle\left\langle\left\langle\left(\int_{f_{L}}^{f_{\mathrm{H}}} S(f) \mathrm{df}\right)^{2}\right\rangle\right\rangle_{A}\right\rangle\right\rangle_{f}\right\rangle_{\mathrm{tr}} \\
& =\int_{f_{L}}^{f_{\mathrm{H}}} \int_{f_{L}}^{f_{\mathrm{H}}}\left\langle\left\langle\left\langle S(f) S\left(f^{\prime}\right)\right\rangle_{A}\right\rangle_{f}\right\rangle_{N_{\mathrm{tr}}} \mathrm{df} \mathrm{df}^{\prime} .
\end{aligned}
$$

The evaluation of the above equation leads to the normalized standard deviation of the noise power spectral density in the frequency band between $f_{L}$ and $f_{H}$

$$
\begin{aligned}
\frac{\sigma_{\mathrm{np}}}{\left\langle n p_{\mathrm{BW}}\right\rangle}= & \frac{\sqrt{\left\langle n p_{\mathrm{BW}}^{2}\right\rangle-\left\langle n p_{\mathrm{BW}}\right\rangle^{2}}}{\left\langle n p_{\mathrm{BW}}\right\rangle} \\
= & \frac{2}{\pi \sqrt{N_{\mathrm{dec}} \mathrm{WL}}} \sqrt{\frac{\left\langle A^{4}\right\rangle}{\left\langle A^{2}\right\rangle^{2}}} \\
& \times \sqrt{\frac{\int_{f_{\mathrm{H}}}^{f_{L} f_{f_{L}}} \frac{1}{\left(f^{\prime 2}-f^{2}\right)} \ln \left(\frac{f^{\prime}}{f}\right) d f d f^{\prime}}{\ln ^{2} \frac{f_{\mathrm{H}}}{f_{L}}}} .
\end{aligned}
$$

Here, the integral in the above equation has no known analytical solution.

For circuit simulation purposes, further simplification is mandatory. If we simplify $\left\langle n p_{\mathrm{BW}}^{2}\right\rangle$ to

$$
\begin{aligned}
& \left\langle n p_{\mathrm{BW}}^{2}\right\rangle \\
& =\int_{f_{L}}^{f_{\mathrm{H}}} \int_{f_{L}}^{f_{\mathrm{H}}}\langle S(f)\rangle\left\langle S\left(f^{\prime}\right)\right\rangle d f d f^{\prime} \\
& =\int_{f_{L}}^{f_{\mathrm{H}}} \int_{f_{L}}^{f_{\mathrm{H}}}\left\langle\sum_{i=1}^{N_{\mathrm{tr}}} \frac{A_{i}^{2}}{f_{i}} \frac{1}{1+\left(\frac{f}{f_{i}}\right)^{2}}\right\rangle \\
& \quad \times\left\langle\sum_{\mathrm{j}=1}^{N_{\mathrm{tr}}} \frac{A_{\mathrm{j}}^{2}}{f_{\mathrm{j}}} \frac{1}{1+\left(\frac{f^{\prime}}{f_{\mathrm{j}}}\right)^{2}}\right\rangle d f d f^{\prime} .
\end{aligned}
$$

The statistical variance in noise power due to scattering in $A_{i}, f_{i}$, and $N_{\text {tr }}$ can be calculated

$$
\begin{aligned}
\sigma_{\mathrm{np}}^{2} & =\left\langle n p_{\mathrm{BW}}^{2}\right\rangle-\left\langle n p_{\mathrm{BW}}\right\rangle^{2} \\
& =\left\langle A^{4}\right\rangle N_{\mathrm{dec}} \mathrm{WL}\left(\frac{\pi}{2} \ln \left(\frac{f_{\mathrm{H}}}{f_{L}}\right)\right)^{2} .
\end{aligned}
$$

The normalized standard deviation is then

$$
\frac{\sigma_{\mathrm{np}}}{\left\langle n p_{\mathrm{BW}}\right\rangle}=\frac{1}{\sqrt{N_{\mathrm{dec}} \mathrm{WL}}} \sqrt{\frac{\left\langle A^{4}\right\rangle}{\left\langle A^{2}\right\rangle^{2}}} .
$$

This simplified equation for the normalized standard deviation of the noise power in the bandwidth of interest shows the same dependency on geometry, average number of traps and RTS amplitude as the exact (64). The dependence on bias point is also the same in both equations. The major difference is the term including the integral in the second square root which cannot be analytically solved. Numerical analysis was performed and this term shows a weak dependency on $f_{L}$ and $f_{H}$. The standard deviation slightly decreases as circuit bandwidth increases. However, the numerical analysis shows that (67) is a good approximation for (64). Equation (67) slightly overestimates the exact value given by (64). Nevertheless, (67) is appropriate for circuit simulation purposes, since it correctly describes the dependency on geometry, average number of traps and RTS amplitude ${ }^{3}$.

For the circuit design it is important to take into account that the standard deviation depends on number of traps, device geometry and bias point, and that the bandwidth dependence is weak.

\section{Contribution of Technology Dependent Long Range Statistical Parameters on the Standard Deviation of LF-Noise}

Equation (67) assumes that there is no correlation between the standard deviation and the spacing $D$ of two devices. However, experimental data reveal correlation between noise amplitude and transistor position, as shown in Fig. 8. The long range correlation distance is considered by a parameter $S$ in the following. This parameter describes the variation of the LF-noise as a function of the spacing $D$ [15]. Modification of (67) thus leads to

$$
\frac{\sigma_{\mathrm{np}}^{2}}{\left\langle n p_{\mathrm{BW}}\right\rangle^{2}}=\frac{1}{N_{\mathrm{dec}} \mathrm{WL}} \frac{\left\langle A^{4}\right\rangle}{\left\langle A^{2}\right\rangle^{2}}+S^{2} D^{2} .
$$

The effect of $S$ is important only for large area devices at significant spacing and can be included into a compact model to simulate the long range variation effects.

\section{EXPERIMENTAL}

In this section, the model proposed in the previous section is validated and compared to experimental data from three different CMOS technologies with minimum feature sizes of 0.25 , $\left(t_{\mathrm{ox}}=5 \mathrm{~nm}, V_{\mathrm{dd}}=2.5 \mathrm{~V}\right), 0.13,\left(t_{\mathrm{ox}}=2.2 \mathrm{~nm}, V_{\mathrm{dd}}=1.5 \mathrm{~V}\right)$, and $0.09 \mu \mathrm{m}\left(t_{\mathrm{ox}}=1.6 \mathrm{~nm}, V_{\mathrm{dd}}=1.2 \mathrm{~V}\right)$.

The average number of traps per transistor $\left\langle N_{\text {tr }}\right\rangle$ is extracted from the average noise behavior using (23), [1] and confirmed by the oxide trap density determined from the LF-noise measured on large area devices. For the $0.25-\mu \mathrm{m}$ technology, the number of traps $N_{\text {tr }}$ is also extracted by performing charge pumping measurements. Reasonable agreement between $N_{\mathrm{tr}}$ evaluated from LF-noise and charge-pumping data is found confirming the proposed strategy.

Furthermore, as seen in Fig. 1, for the smallest devices the Lorentzian shape of individual RTS dominates the LF-noise characteristics, and a lower limit for number of traps $N_{\mathrm{tr}}$ is estimated by counting the visible Lorentz curves superposed in the measured frequency range. This method may underestimate

${ }^{3}$ The reader is strongly encouraged to contact the authors on the details of the derivations of the model equations. To explicitly show all derivations would lead to an inappropriately large amount of equations and will be published elsewhere 
TABLE I

AVERAGe Number of Traps Per Transistor $\left\langle N_{\mathrm{tr}}\right\rangle,\left(N_{\mathrm{dec}} * W L\right)^{-0.5}$ AND $\left(\left\langle A^{4}\right\rangle /\left\langle A^{2}\right\rangle^{2}\right)^{0.5}$. DATA IS From MinimUM AREA TRANSISTORS OF

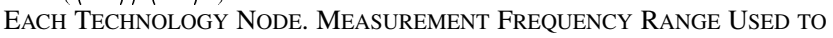
Evaluate $\left\langle N_{\mathrm{tr}}\right\rangle$ AND $N_{\text {dec }}$ Is $1 \mathrm{~Hz}$ TO $10 \mathrm{kHz}$

\begin{tabular}{c|c|c|c|c|c}
\hline $\begin{array}{c}\text { Technology } \\
\text { Node }\end{array}$ & $\begin{array}{c}W \times L \\
(\mu \mathrm{m} \times \mu \mathrm{m})\end{array}$ & $\begin{array}{c}\sigma_{n p} \\
\text { Normalized }\end{array}$ & $\left\langle N_{t r}>\right.$ & $\left(N_{d e c}{ }^{*} W L\right)^{-0.5}$ & $\left(\left\langle A^{4}\right\rangle /\left\langle A^{2}\right\rangle^{2}\right)^{0.5}$ \\
\hline $0.25 \mu \mathrm{m}$ & $0.30 \times 0.25$ & 1.87 & $9^{*}$ & 1.01 & 1.85 \\
\hline $0.13 \mu \mathrm{m}$ & $0.16 \times 0.13$ & 3.57 & $2.7^{+}$ & 1.85 & 1.93 \\
\hline $0.09 \mu \mathrm{m}$ & $0.12 \times 0.09$ & 3.43 & $1.8^{+}$ & 2.26 & 1.52 \\
\hline
\end{tabular}

* Extracted from charge pumping measurements and LF-noise data.

+ Extracted from LF-Noise data.

$N_{\text {tr }}$, since Lorentzians with smaller amplitudes can be missed, and is valid only to set a lower limit on $N_{\text {tr }}$ to verify the results. Nevertheless, $\left\langle N_{\mathrm{tr}}\right\rangle$ extracted from average noise behavior is found to be within the estimated limit and is consistent with charge pumping data [1].

After the extraction of $N=\left\langle N_{\mathrm{tr}}\right\rangle, 1 / N_{\mathrm{dec}}$ is calculated using (28) (Table I). The term $\left(\left\langle A^{4}\right\rangle /\left\langle A^{2}\right\rangle^{2}\right)^{0.5}$ can then be evaluated. The resulting variations of the normalized noise amplitude are higher than expected for the case where only number fluctuations in $N_{\mathrm{tr}}$ are taken into account, since $N_{\mathrm{tr}}$ is expected to be Poisson distributed and for a Poisson distribution $\sigma_{N_{\mathrm{tr}}} /\left\langle N_{\mathrm{tr}}\right\rangle=$ $\left\langle N_{\mathrm{tr}}\right\rangle^{-0.5}$ is expected [28]. This fact experimentally confirms that RTS-Amplitude variations are relevant for the statistical variations of the LF-noise amplitude. Strong amplitude variations in Lorentzians with similar corner frequencies found in devices with similar geometry also corroborate the relevance of RTS-Amplitude variations.

Fig. 5 shows the normalized standard deviation of the LF-noise of measured transistors as a function of device area. The area dependence predicted by (67) and (68) is clearly observed here showing excellent agreement between experiment and model.

In long-channel devices, the average gate referred voltage noise is widely independent of bias conditions. However this is not true for noise behavior between different deep-submicrometer devices. As can be seen in Fig. 2, strong variations in noise performance do not only appear between different devices, but also for different bias points of a single device. Generally, variations in noise amplitude increase for large gate and especially drain voltages due to the increasing influence of the trap position in the channel, as seen in Fig. 6, where the mean values of all experimental data points show a trend toward higher variance with increasing drain voltage. Model and experiment show agreement within the accuracy of the measurements (without any technology dependent additional fit parameter).

Another important conclusion drawn from model and experimental data is the strong asymmetry of the statistical distribution of the noise power spectral density of small area devices. Fig. 7 shows the histogram of the distribution of integrated noise power of devices from the $0.13-\mu \mathrm{m}$ technology node. The distribution is clearly asymmetrical for the smallest devices. Furthermore, Table I, Figs. 5 and 6 show that for the smallest devices the normalized standard deviation is greater than one, i.e., for these devices the standard deviation is greater than the average value. This does not mean that one can get "negative"

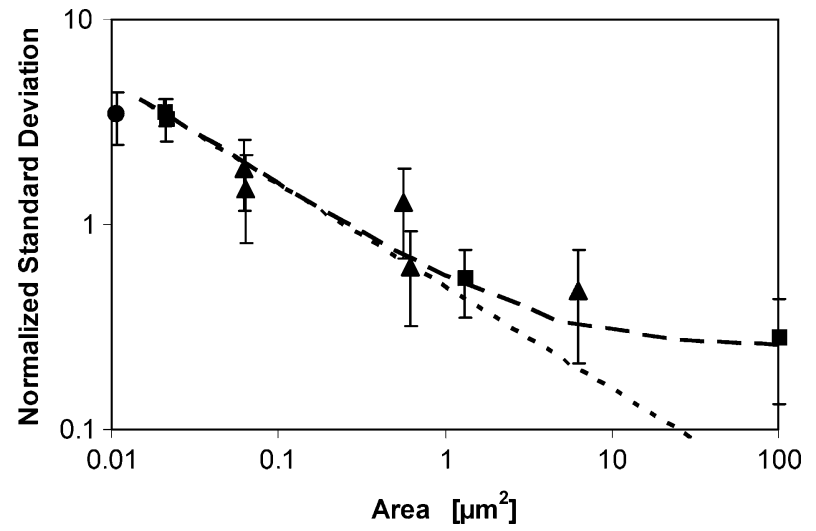

Fig. 5. Normalized standard deviation of gate referred voltage noise integrated in the bandwidth $1 \mathrm{~Hz}$ to $10 \mathrm{kHz}$ versus area for transistors biased in saturation. Error bars are $2 \sigma$-values of the measurement accuracy. $\boldsymbol{\Delta} 0.25-\mu \mathrm{m}$ technology node $\left(L_{\min }=0.25 \mu \mathrm{m}, t_{\mathrm{ox}}=5 \mathrm{~nm}\right)$. Total of 30 transistors measured. $0.13-\mu \mathrm{m}$ technology node $\left(L_{\min }=0.13 \mu \mathrm{m}, t_{\mathrm{ox}}=2.2 \mathrm{~nm}\right)$. Total of 127 transistors measured. $\bullet 90-\mathrm{nm}$ technology node $\left(L_{\min }=0.09 \mu \mathrm{m}, t_{\mathrm{ox}}=\right.$ $1.6 \mathrm{~nm})$. Total of 14 transistors measured. The dashed line shows results for the $0.13-\mu \mathrm{m}$ node calculated using (68). The dotted line is a guide line of slope -0.5 . (Normalized standard deviation is standard deviation of the square of integrated gate referred voltage noise divided by the average of the square of integrated gate referred voltage noise).

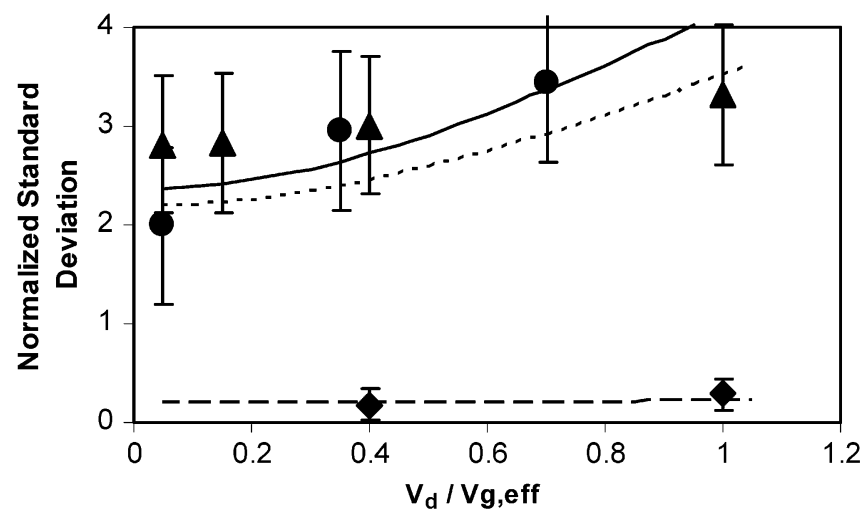

Fig. 6. Normalized standard deviation of the gate referred voltage noise versus drain voltage for $W / L=0.16 \mu \mathrm{m} / 0.13 \mu \mathrm{m}$ p-MOS transistors operated at $\left|V_{g}-V_{\mathrm{th}}\right|=0.60 \mathrm{~V}(\boldsymbol{\Delta}), W / L=10 \mu \mathrm{m} / 10 \mu \mathrm{m} \mathrm{n}-\mathrm{MOS}$ transistors at $V_{g}$ $V_{\mathrm{th}}=0.60 \mathrm{~V}(\bullet)$ and $W / L=0.12 \mu \mathrm{m} / 0.09 \mu \mathrm{m} \mathrm{n}$-MOS transistors at $V_{g}-V_{\mathrm{th}}=0.55 \mathrm{~V}(\bullet)$. The full line shows the result of a calculation based on the model (55) and (68) for $W / L=0.12 \mu \mathrm{m} / 0.09 \mu \mathrm{m}$ n-MOS transistors (90-nm technology - all other data from $0.13-\mu \mathrm{m}$ technology), the dashed line for the $W / L=10 \mu \mathrm{m} / 10 \mu \mathrm{m} \mathrm{n}$-MOS transistors, and dotted line for the $W / L=0.16 \mu \mathrm{m} / 0.13 \mu \mathrm{m}$ p-MOS transistors.

noise power, but that the distribution is asymmetrical. Since $N_{\text {tr }}$ is Poisson distributed, a strongly asymmetrical distribution for small $\left\langle N_{\mathrm{tr}}\right\rangle$ is expected. For distributions whose actual shape is not known, Chebyshevs inequality can be used to estimate confidence intervals [28]. The Chebyshev inequality states that for a $k>1$ the proportion of observations which lie within $k$ standard deviations of the mean is at least $\left(1-1 / k^{2}\right)$, regardless of the shape of the distribution. This means, for instance, that at least $88.89 \%$ of the observations are expected to lie within $3 \sigma$ from the mean value. Note that in the case of a Gaussian distribution a higher amount of the observations would be expected to lie within the $3 \sigma$ interval. Since the device noise can not be lower than the thermal noise, for practical purposes the thermal noise could be used as the lower bound of the confidence interval if Chebishevs inequality leads to 

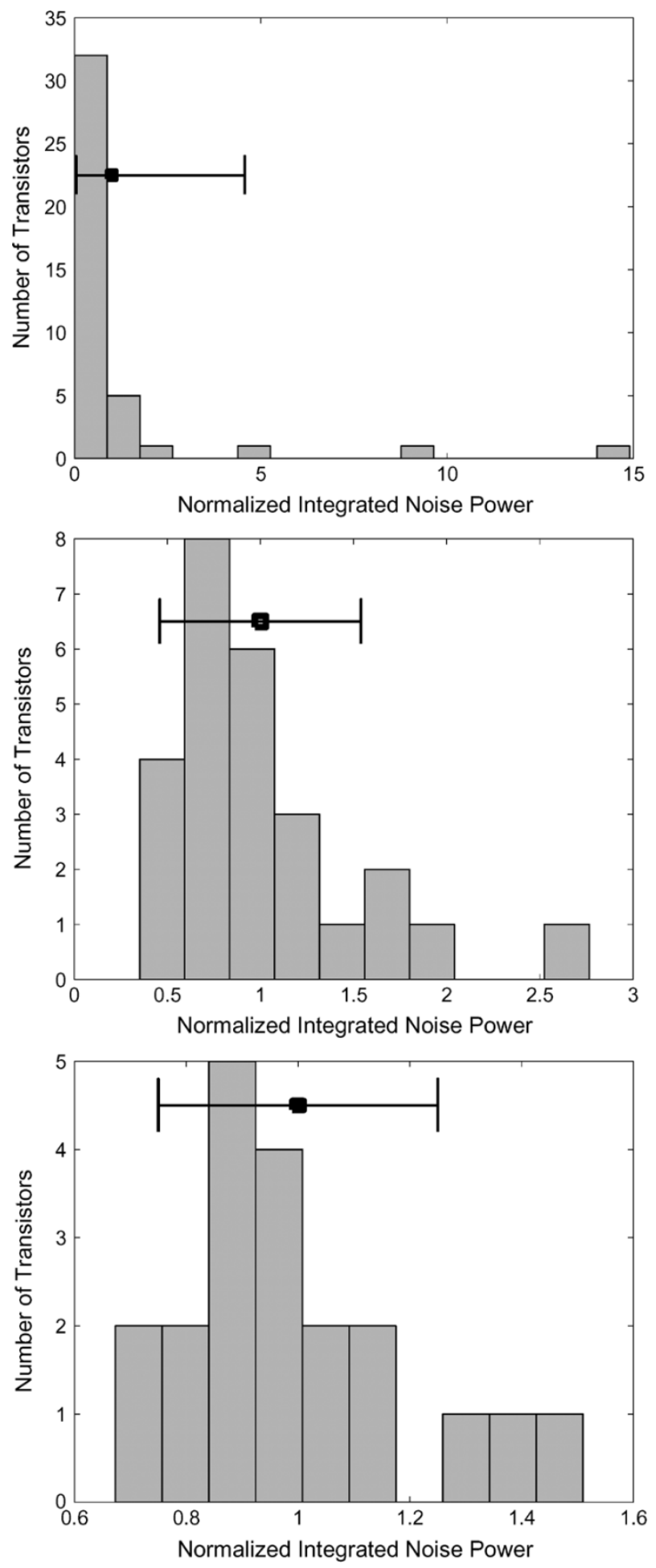

(b)

(c)

Fig. 7. Histogram of the distribution of normalized integrated noise power of transistors with (a) $W=0.16 \mu \mathrm{m}, L=0.13 \mu \mathrm{m}$; (b) $W=10 \mu \mathrm{m}, L=$ $0.13 \mu \mathrm{m}$; and (c) $W=L=10 \mu \mathrm{m}$. The noise data are integrated from 1 $\mathrm{Hz}$ to $10 \mathrm{kHz}$ for transistors biased in saturation. Normalized integrated noise power is integrated noise power of a transistor divided by the average value of integrated noise power of transistors with the same geometry. Squares indicate average value and error bars show the standard deviation. Since noise power can not become negative, the thermal noise is used as the lower bound for transistors in (a). The average value of normalized integrated noise power is always one.

a confidence interval with a smaller lower bound. The upper boundary of the confidence interval can always be evaluated

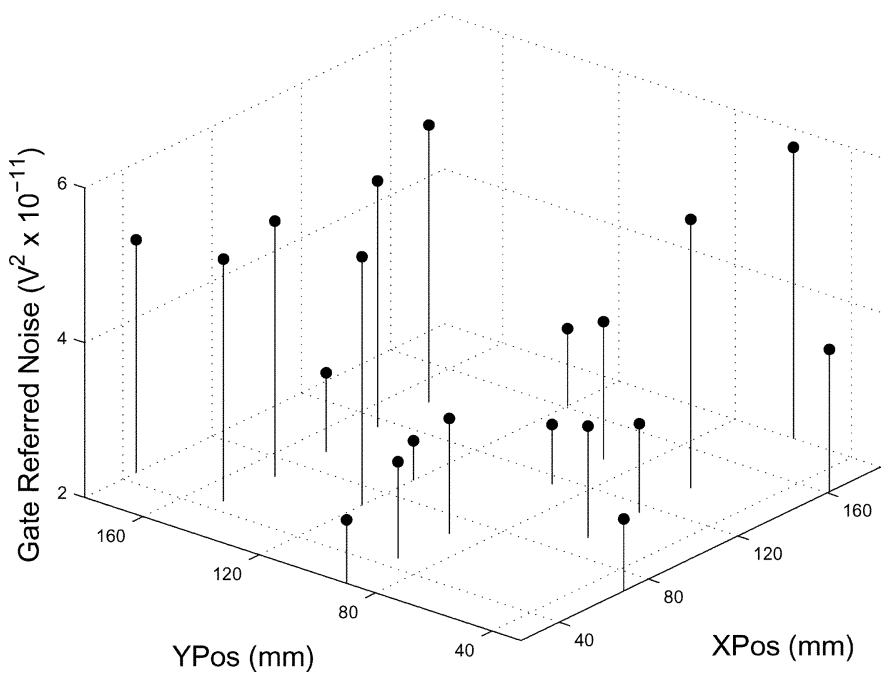

Fig. 8. Gate referred voltage noise (integrated bandwidth $1 \mathrm{~Hz}-10 \mathrm{kHz}$ ) of $20 \mathrm{n}$-MOSFETs from a $0.13-\mu \mathrm{m}$ standard CMOS process with $t_{\mathrm{ox}}=2.2$ $\mathrm{nm}$, at different wafer positions. Device dimensions are $W=L=10 \mu \mathrm{m}$, characterization is performed in saturation at $V_{g}-V_{\mathrm{th}}=0.25 \mathrm{~V}$ and $V_{d}=1.0$ $\mathrm{V}$. $\mathrm{X}$ and $\mathrm{Y}$ axis indicate die $x$ and $y$ position in millimeters, on a $200 \mathrm{~mm}$ wafer. The measurements have been performed on a test wafer. Therefore, not all positions show target device data. These positions are not included here.

using Chebyshevs inequality together with the model equations. For most distributions Chebyshevs inequality is very conservative and tighter confidence intervals may be evaluated if the actual shape of the distribution is known. The final statistical distribution of noise power spectral density is the result of the complex interplay of the distributions of $N_{\mathrm{tr}}, f_{i}$, and $A_{i}$ as given by (30). Theoretical evaluation and experimental verification of the noise power spectral density distribution is relevant work that has to be undertaken to allow the determination of tighter confidence intervals. If the variation of noise level has a log-normal distribution, a logarithmic scale may be used in the evaluation of average value and standard deviation [8]-[10], avoiding negative values for the worst case parameters. In this case the properties of the normal distribution may be used in the estimation of confidence intervals. Another approach found in the literature is the use of geometric mean of power spectra in the evaluation of average value and standard deviation [30].

The distribution of average integrated noise power (bandwidth $1 \mathrm{~Hz}$ to $10 \mathrm{kHz}$ ) of large area devices across a 200-mm wafer is shown in Fig. 8. This plot gives an idea about long range process related variations. Analysis of long range noise amplitude variation versus distance for the 0.25 and $0.13-\mu \mathrm{m}$ nodes leads to SD equal to 0.18 and 0.26 [see (68)], respectively.

\section{CONCLUSION}

This paper included analytical and statistical modeling, device simulations and experimental results for the LF-noise behavior in deep-submicrometer MOSFETs. The impact of statistical effects on the LF-noise performance of CMOS devices in modern technologies was discussed. A novel LF-noise model including detailed physics based modeling of statistical effects was presented. The model derivation was in strong relation with the device physics and was consistent with MOSFET scaling. 
Strong variations of noise performance may appear not only between devices, but also for a single device operated under different bias conditions. The noise performance is shown to depend on the number of traps, the trap position within the channel, on the depth of the trap location within the oxide, on the bias point, on device geometry, and on long-range statistical parameters.

The statistical model is based on microscopic instead of distributed quantities, and is compatible with BSIM or related compact models.

\section{REFERENCES}

[1] R. Brederlow, W. Weber, D. Schmitt-Landsidel, and R. Thewes, "Fluctuations of the low frequency noise of MOS transistors and their modeling in analog and RF-circuits," in IEDM Tech. Dig., 1999, pp. 159-162.

[2] T. Boutchacha and G. Ghibaudo, "Low frequency noise characterization of $0.18 \mu \mathrm{m}$ Si CMOS transistors," Phys. Statist. Sol. (a), vol. 167, pp. 261-270, May 1998.

[3] G. Ghibaudo and O. Roux-dit-Buisson, "Low-frequency fluctuations in scaled-down silicon CMOS devices: Status and trends," in Proc. ESSDERC, 1994, pp. 693-700. Editions Frontiers.

[4] G. Ghibaudo, O. Roux-dit-Buisson, and J. Brini, "Impact of scaling down on low frequency noise in silicon MOS transistors," Phys. Statist. Sol. A, vol. 132, pp. 501-507, 1992.

[5] M. H. Tsai and T. P. Ma, "The impact of device scaling on the current fluctuations in MOSFETs," IEEE Trans. Electron Devices, vol. 41, no. 11, pp. 2061-2068, Nov. 1994.

[6] H. M. Bu, Y. Shi, Y. D. Zheng, S. H. Gu, H. Majima, H. Ishikuro, and T. Hiramoto, "Impact of the device scaling on the low-frequency noise in n-MOSFETs," Appl. Phys. A, vol. A71, pp. 133-136, 2000.

[7] P. K. Hurley, S. Moran, L. Wall, A. Mathewson, and B. Mason, "Mechanics of the low frequency noise in P channel MOSFETs," in Proc. ESSDERC, 1994, pp. 147-150. Editions Frontiers.

[8] M. J. Deen, O. Marinov, D. Onsongo, and S. Banerjee, "Low-frequency noise in SiGeC-based pMOSFETs," Proc. SPIE, vol. 5470, pp. 215-225, 2004.

[9] M. Sánden, O. Marinov, M. J. Deen, and M. Ostling, "A new model for the low-frequency noise and the noise level variation in polysilicon emitter BJTs," IEEE Trans. Electron Devices, vol. 49, no. 3, pp. 514-519, Mar. 2002.

[10] emitter bipolar junction transistors," IEEE Electron Device Lett., vol. 22, pp. 242-244, May 2001.

[11] R. Jayaraman and C. G. Sodini, "A $1 / f$ noise technique to extract the oxide trap density near the conduction band edge of silicon," IEEE Trans. Electron Devices, vol. 36, no. 9, pp. 1773-1782, Sep. 1989.

[12] E. Simoen, B. Dierickx, C. L. Clayes, and G. J. Declerck, "Explaining the amplitude of RTS noise in submicrometer MOSFETs," IEEE Trans. Electron Devices, vol. 39, no. 2, pp. 422-419, Feb. 1992.

[13] A. Godoy, F. Gámiz, A. Palma, J. A. Jiménez-Tejada, J. Banqueri, and J. A. López-Villanueva, "Influence of mobility fluctuations on random telegraph signal amplitude in n-channel metal-oxide-semicondutor fieldeffect transistors," J. Appl. Phys., vol. 82, pp. 4621-4628, Nov. 1997.

[14] P. Stolk, F. P. Widdershoven, and D. B. M. Klaassen, "Modeling statistical dopant fluctuations in MOS transistors," IEEE Trans. Electron Devices, vol. 45, no. 9, pp. 1960-1971, Sep. 1998.

[15] U. Schaper, C. G. Linnenbank, and R. Thewes, "Precise characterization of long-distance mismatch of CMOS devices," IEEE Trans. Semcond. Manuf., vol. 14, pp. 311-317, Nov. 2001.

[16] G. Wirth, U. Hilleringmann, J. T. Horstmann, and K. Goser, "Negative differential resistance in ultrashort bulk MOSFETs," in Proc. IEEE Industrial Electronics Society, 1999, pp. 29-34.

[17] MiniMos Manual (2004). [Online]. Available: http:// www.iue.tuwien.ac.at/

[18] P. Dutta and P. M. Horn, "Low-frequency fluctuations in solids: $1 / f$ noise," Rev. Mod. Phys., vol. 53, pp. 497-516, Jul. 1981.

[19] M. J. Kirton and M. J. Uren, "Noise in solid-state microstructures: A new perspective on individual defects, interface states and low-frequency $1 / f$ noise," Adv. Phys., vol. 38, pp. 367-468, 1989.

[20] S. Christensson, I. Lundström, and C. Svensson, "Low frequency noise in MOS transistors-I theory," Solid State Electron., vol. 11, pp. $791-812,1968$.
[21] G. Reimbold, "Modified $1 / f$ trapping noise theory and experiments in MOS transistors from weak to strong inversion-influence of interface states," IEEE Trans. Electron Devices, vol. 31, no. 9, pp. 1190-1998, Sep. 1984.

[22] S. M. Sze, Physics of Semiconductor Devices, 2nd ed. New York: Wiley, 1981.

[23] BSIM4 Manual (2001). [Online]. Available: http://www-device.EECS.Berkeley.EDU/ bsim4/

[24] S. Villa, A. Lacaita, L. Perron, and R. Bez, "A physically-based model of the effective mobility in heavily-doped n-MOSFETs," IEEE Trans. Electron Devices, vol. 45, no. 1, pp. 110-115, Jan. 1998.

[25] S. T. Martin, G. P. Li, and J. Worley, "The gate bias and geometry dependence of random telegraph signal amplitudes," IEEE Electron Device Lett., vol. 18, pp. 444-446, Sep. 1997.

[26] R. Brederlow, "Niederfrequentes rauschen in analogen CMOS schaltungen," Ph.D. dissertation, Dept. Elect. Eng., Tech. Univ. Berlin, Berlin, Germany, 1999.

[27] K. K. Hung, P. K. Ko, C. Hu, and Y. C. Cheng, "A unified model for the flicker noise in metal-oxide-semiconductor field-effect transistors," IEEE Trans. Electron Devices, vol. 37, no. 3, pp. 654-665, Mar. 1990.

[28] I. N. Bronstein and K. A. Semendjajew, Taschenbuch der Mathematik, Teubner Verlagsgesellschaft, Leipzig, 1989.

[29] A. P. van der Wel, E. A. M. Klumperink, L. K. J. Vandamme, and B Nauta, "Modeling random telegraph noise under switched bias conditions using cyclostationary RTS noise," IEEE Trans. Electron Devices, vol. 50, no. 5, pp. 1378-1384, May 2003.

[30] R. Pintelon, J. Schoukens, and J. Renneboog, "The geometric mean of power (amplitude) spectra has a much smaller bias than the classical arithmetic (RMS) averaging," IEEE Trans. Instrum. Meas., vol. 37, no. 6, pp. 213-218, Jun. 1988.

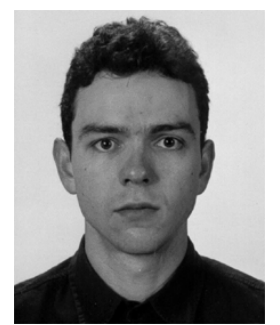

Gilson I. Wirth (M'97) received the B.S.E.E. and M.Sc. degrees from the Federal University of Rio Grande do Sul (UFRGS), Brazil, in 1990 and 1994, respectively, and the Dr.-Ing. degree in electrical engineering from the University of Dortmund, Dortmund, Germany, in 1999.

From 2000 to 2002, he was a Lecturer and Researcher in the field of microelectronics at the Informatics Institute, UFRGS. In 2002, he joined the Computer Engineering Department, State University of Rio Grande do Sul, where he is currently a Professor and Head of the research group in micro- and nano-electronics. In July, August, and December 2001, he was with Motorola, Austin, TX, working on CMOS process technology transfers to CEITEC, Porto Alegre, Brazil. In February and March 2002, he was with the Corporate Research Department, Infineon Technologies, Munich, Germany, working as Guest Researcher on LF-noise in deep-submicrometer MOS devices. His research interests include LF-noise, device modeling, and analog and mixed-signal circuit design.

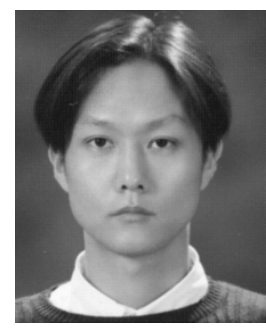

Jeongwook Koh was born in Seoul, Korea in 1975. $\mathrm{He}$ received the M.Sc. in electronics engineering from Sogang University, Seoul, Korea, in 2001, and the M.S. degree in the field of the electrothermal modeling of a MOSFET, and the Dr.-Ing. degree as part of a cooperative program between Technical University of Munich, Munich, Germany, and Corporate Research, Infineon Technologies AG, Munich, in 2000 and 2005, respectively. As part of his doctoral research, he developed a novel $1 / \mathrm{f}$ noise reduction technique suitable for linear analog CMOS

circuits.

In 2000, he was with the Power Device Division, Infineon Technologies AG, Munich, in the field of electrothermal modeling of a MOSFET. During that period he developed a novel $1 / f$ noise reduction technique for linear analog CMOS circuits. He is currently with the Communication and Network Laboratory, Samsung Advanced Institute of Technology, Samsung Electronics Company, Ltd., Kiehung, Korea, where he is responsible for the analog CMOS baseband circuitries for wireless LAN applications. His research interests include the LF-noise phenomena in modern CMOS technologies and the related analog CMOS IC design with special emphasis on low LF-noise. 


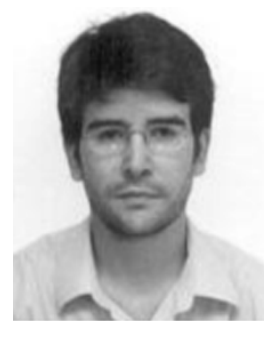

Roberto da Silva was born in Mauá, São Paulo, Brazil, in 1973. He received the B.S. and Ph.D. degrees in physics from the University of São Paulo in 1997 and 2002, respectively.

From 1997 to 2002, he worked on statistical mechanics, mathematical physics, and computational physics where most of his papers are published in indexed journals. Since 2003, he is Professor at the Federal University of Rio Grande do Sul, Porto Alegre, Brazil. His research interests include numerical and statistical modeling in scientific computing.

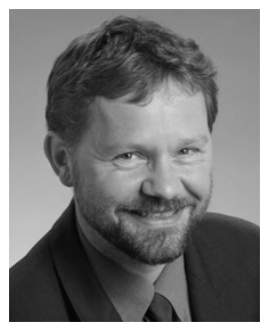

Roland Thewes (M'00) was born in Marl, Germany, in 1962. He received the Dipl.-Ing. and Dr.-Ing. degrees in electrical engineering from the University of Dortmund, Dortmund, Germany, in 1990 and 1995, respectively.

From 1990 to 1995, he was part of a cooperative program between Siemens Research Laboratories, Munich and the University of Dortmund in the field of hot-carrier degradation in analog CMOS circuits. Since 1994, he has been with the Research Laboratories, Infineon Technologies AG, where he has been active in the design of nonvolatile memories and in the field of reliability and yield of analog CMOS circuits. From 1997 to 1999, he managed projects in the fields of design for manufacturability, reliability, analog device performance, and analog circuit design. Since 2000, he is responsible for the Advanced Mixed-Signal Circuits Laboratory, Corporate Research, Infineon Technologies. His current interests include electronic bio-sensors on CMOS, device physics-related circuit design, and advanced analog CMOS circuit design. He has authored or coauthored some 100 publications including book chapters, tutorials, invited papers, etc., and he is giving lectures at the University of Ulm, Ulm, Germany.

Dr. Thewes is a member of the German Association of Electrical Engineers (VDE). He served as a member of the technical program committees of the International Reliability Physics Symposium (IRPS), and of the European Symposium on Reliability of Electron Devices, Failure Physics and Analysis (ESREF). He is a member of the technical program committees of the International Solid-State Circuits Conference (ISSCC), of the International Electron Device Meeting (IEDM), and of the European Solid State Device Research Conference (ESSDERC). Moreover, in 2004, he joined the IEEE EDS VLSI Technology and Circuits Committee.

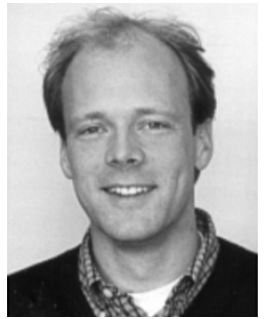

Ralf Brederlow was born in Munich, Germany, in 1970. He received the Dipl.-Phys. degree from the Technical University of Munich, Munich, Germany, in 1996 and the Dr.-Ing. degree from the Technical University of Berlin, Berlin, Germany, in 1999.

From 1995 to 1996 , he worked at the WalterSchottky Institute, Garching, Germany, in the field of $\mathrm{Si} / \mathrm{SiGe}$ intersub-band detectors and $\mathrm{Si} / \mathrm{SiGe}-\mathrm{MBE}$. In 1996 he joined a cooperative program between Siemens Corporate Research, Munich, and the Technical Universities of Munich and Berlin, where he worked on the characterization, modeling, and reliability of noise in analog circuits. 1999 he joined the Corporate Research Department of Infineon Technologies. Since then he has worked on many projects in the field of technology related circuit design, including noise, and mismatch modeling, device reliability, polymer electronics, and sensors for bio-chemical applications. His current work is focused on noise-tolerant and reliable analog and digital circuit design. He has authored or coauthored some 40 technical publications and holds several patents.

$\mathrm{He}$ is a member of the Program Committee International Electron Device Meeting (IEDM) and is a member of the Design Technical Working Group and the RF and analog Technologies for Wireless Communication Technical Working Group of the International Technology Roadmap for Semiconductors (ITRS). 